\title{
STUDYING SWIMMING VELOCITY AND RHEOTAXIS IN RELATION TO SPERM COMPETITION AND IN VIVO SURVIVAL IN EGYPTIAN LOCAL CHICKENS
}

\author{
M.A.M. Sayed, M.W. Marzouk, T.M. El-Sherry and O. Afifi
}

Department of Poultry Production, Faculty of Agriculture, Assiut University, Assiut, Egypt. 71515

In the current trial, we studied sperm velocity, progressive motility and the percentage of sperms swimming against the flow (rheotaxis) in two Egyptian local chicken strains (Sharkasi and Dandarawi) in relation to sperm competition and sperm survival within the oviduct capable of fertilizing the ova.

A total number of 5 males and 20 females, 30 weeks of age, from each strain were used. For studying sperm competition, semen was collected from all males and pooled within each strain and sperm concentration was assessed using a hemocytometer. Afterwards, semen pools were mixed in which the final semen mixture was having an equal number of spermatozoa from each strain. Ten females from each strain were artificially inseminated using the final semen pool. Artificial insemination was repeated ten times (once every three days). Fertile eggs from each strain were collected, marked and incubated and the hatching chicks were distinguished as coming from Sharkasi or Dandarawi fathers according to their phenotype. For assessing in vivo sperm survival, ten females were artificially inseminated by semen donated from males of the same strain. Eggs were collected for a period of 22 days and incubated to find out the fertility period. The results showed that ejaculates from Sharkasi roosters had sperms with higher velocity and higher number of rheotactic spermatozoa compared to those of Dandarawi roosters. Incubation of fertile eggs collected from Sharkasi females resulted in 111 hatching chicks descending from Sharkasi fathers and 25 chicks from Dandarawi males. Similarly, fertile eggs laid by Dandarawi mothers produced 88 chicks descending from Sharkasi fathers and 42 chicks from Dandarawi males. Finally, in vivo stored Sharkasi spermatozoa were capable of fertilizing the ova after 22 days of the insemination which was markedly different than that of Dandarawi which produced fertile eggs until 14 days of the insemination. It can be concluded that sperm velocity and rheotaxis can be used to predict the sperm competence and its ability to survive within the sperm storage tubules. 